\title{
From graphite to laccase biofunctionalized few-layer graphene: a "one pot" approach using a chimeric enzyme
}

\author{
Ilaria Sorrentino ${ }^{1}$, Ilaria Stanzione ${ }^{2}$, Yannig Nedellec ${ }^{1}$, Alessandra Piscitelli $^{2}$, Paola \\ Giardina $^{3}$, and Alan le goff ${ }^{1}$ \\ ${ }^{1} \mathrm{CNRS}$ \\ ${ }^{2}$ Universita degli Studi di Napoli Federico II Complesso Universitario di Monte SantÁngelo \\ ${ }^{3}$ University of Naples
}

May 5, 2020

\begin{abstract}
A chimeric enzyme based on the genetic fusion of a laccase with a hydrophobin domain was employed to functionalize few-layergraphene, previously exfoliated from graphite in the presence of the hydrophobin. The as-produced biofunctionalized FLG was characterized by electrochemistry and Raman spectroscopy and finally employed in the biosensing of phenols such as catechol and dopamine. This strategy paves the way for the functionalization of nanomaterials by hydrophobin domains of chimeric enzymes and their use in a variety of electrochemical applications.
\end{abstract}

KEYWORDS: laccase, hydrophobin, graphene, chimeric enzymes, biosensing INTRODUCTION

Graphene is a two-dimensional sheet of $\mathrm{sp}^{2}$-hybridized carbon that exhibits unparalleled properties such as high planar surface, superlative mechanical strength, and remarkable thermal and electrical conductivity. Due to its extraordinary structure and fascinating properties, graphene is the most studied nanomaterial and can be integrated as the core of cutting-edge devices in many types of applications, ranging from microelectronics to electrochemical energy harvesting systems (Brownson, Lacombe, Gómez-Mingot, \& Banks, 2011; Brownson, Munro, Kampouris, \& Banks, 2011; C. Ferrari et al., 2015; Kampouris \& Banks, 2010; Novoselov et al., 2012; Pumera, 2012). In biosensing, especially, graphene act as a conductive platform for biomolecules immobilization and electrochemical detection of bioanalytes (Fritea et al., 2015; Fritea, Tertis, Sandulescu, \& Cristea, 2018; Lalaoui, Le Goff, Holzinger, Mermoux, \& Cosnier, 2015; Le Goff, Reuillard, \& Cosnier, 2013; Pumera, 2011; Zhang, Jia, \& Furumai, 2018). Graphene-based electrochemical biosensing has relied on the recent developments in the study of graphene electrochemical properties, its production and biofunctionalization. Different techniques have been investigated for the production of graphene such as scotch-tape transfer, Chemical Vapour Deposition (CVD) growth and chemically- or electrochemicallyreduced graphene oxide. These strategies lead to different nanomaterials in terms of size, edge and basal defects, number of layers and oxygenated defect content. While CVD produces a large surface of monolayer graphene, soft exfoliation of graphite has also been able to provide low-cost access to few-layer-graphene (FLG) dispersions. The dispersion stability is one of the main challenges to address during the exfoliation procedure, in general, the re/aggregation of exfoliated material is minimized by using organic solvents or surfactant-water solutions (Keeley et al., 2011; Lotya et al., 2009; Smith, Lotya, \& Coleman, 2010). As other Nanomaterials, Graphene is a very suitable platform for enzyme immobilization thanks to its high surface area, dispersion in solution, tuneable surface chemistry. Indeed, intense efforts have been devoted to this research field in the last five years, resulting in the immobilization of different enzymes for various 
applications. (B. Ramakrishna, D. Nalder, Yang, N. Marshall, \& J. Barrow, 2018) However, the hydrophobic interactions driving the direct immobilization of active proteins on graphene surface are often difficult to achieve or modifications of the protein 3D structures can occur with detrimental effect on their functionality. (C. Rodrigues, Ortiz, Berenguer-Murcia, Torres, \& Fernandez-Lafuente, 2013) Two main approaches have been implemented- and often combined- to overcome this issue, such as the use of Graphene oxide (GO) whose surface is more hydrophilic, or the exploitation of Graphene based composites, e.g. microcellulose, chitosan, and various metal oxides (B. Ramakrishna et al., 2018).

Recently, proteins prone to form amyloid structures have proven able to biofunctionalize graphene. In this respect, the fungal self-assembling class I hydrophobin Vmh2 has been successfully exploited to disperse and stabilize FLG in ethanol-water mixtures by ultrasonic wave exfoliation. (Gravagnuolo et al., 2015) Hydrophobins (HFB) are a family of small self-assembling proteins produced by filamentous fungi, they can be divided into two classes which differ for the nature of the amphipathic layers that they form. Fibrillar structures formed by class I HFB are extremely robust, are disassembled only in strong acids and share structural properties with amyloid fibrils. (Zampieri, Wosten, \& Scholtmeijer, 2010) HFB efficiently adhere to several hydrophobic surfaces, among those it can be highlighted 2D materials, such as graphene. (Gravagnuolo et al., 2015) This ability has been further exploited by genetic fusion of the hydrophobin to biotechnologically relevant proteins which can be immobilized on various surfaces, obtaining the so called "self-immobilizing" proteins/enzymes. (Piscitelli, Pennacchio, Longobardi, Velotta, \& Giardina, 2017) Recently a new chimeric protein, Lac-Vmh2, was designed to combine the HFB Vmh2 to a laccase enzyme. (Sorrentino, Giardina, \& Piscitelli, 2019) Laccases ( $p$-diphenol-dioxygenoxidoreductases; EC 1.10.3.2) are multicopper oxidases able to catalyze the oxidation of a wide range of aromatic substrates using oxygen as co-substrate and producing water as the only by-product. These enzymes are promising biocatalysts with possible applications in bioremediation, chemical synthesis, biobleaching of paper pulp and biosensing (Pezzella, Guarino, \& Piscitelli, 2015). Laccases have been immobilized on various carriers, using different methods with both advantages and drawbacks. Among the laccases, POXA1b from Pleurotus ostreatus was chosen for its peculiar characteristics such as its stability and activity in a wide range of $\mathrm{pHs}$ and temperatures, as well as its high redox potential.(Pezzella et al., 2017)The produced Lac-Vmh2 allowed achievement of simple and stable immobilization of the enzyme on polystyrene. (Sorrentino et al., 2019)

The main purpose of this work was to step forward the biofunctionalization of Graphene with Vmh2, immobilizing laccase on FLG by using the fusion protein Lac-Vmh2 through a "one-pot" approach. The presented method is easy, eco-friendly and versatile, since, in principle, a wide variety of different HFBchimera proteins can be used in this one-pot exfoliation/functionalization procedure. As a proof of concept, the as-prepared Lac-Vmh2/FLG was used for the modification of GC electrodes to build an electrochemical sensor for phenolic compounds, such as catechol, a well-known environmental pollutant, and dopamine, a renowned neurotransmitter.

\section{MATERIALS AND METHODS}

All products were purchased from Sigma Aldrich and were used without further purification. All solvents were of analytical grade. Distilled water was passed through a Milli-Q water purification system to obtain ultrapure water at $18.2 \mathrm{M} \mathrm{cm}^{-1}$. Phosphate buffer solution (PB) solution was prepared from Milli-Q water.

\section{Electrochemical measurements}

The electrochemical experiments were carried out in a three-electrode electrochemical cell using a Ametek Multipotentiostat Princeton Applied Research. A Pt wire was used as the counter electrode and the Saturated Calomel electrode (SCE) served as the reference electrode. All experiments were conducted at room temperature. All simulated curves were obtained via Origin Pro 9.0. Error bars were estimated from 3 measurements recorded per sample.

\section{Laccase enzymes}

Both fusion proteins Lac-Vmh2 and wild-type enzyme POXA1b were produced and secreted by the yeast 
Pichia pastoris in the culture media. The supernatant, after centrifugation for 15 min at 6,000 rpm at 4 $\operatorname{degC}$, was concentrated and dialyzed towards $50 \mathrm{mM}$ Tris-HCl buffer, $\mathrm{pH}$ 8.0, using Centricon Centrifugal Filter Units 10kDa (Merck, Darmstadt, Germany). The laccase enzymes are used without additional purification steps. The total protein concentration was determined using the Pierce 660 method (Thermo Fischer Scientific) and using BSA as the standard. The laccase activity was assayed at room temperature, monitoring the oxidation of ABTS (2,2'-azino-bis(3-ethylbenzothiazoline-6-sulphonic acid)) at $420 \mathrm{~nm}\left(\varepsilon_{420 \mathrm{~nm}}=3.6\right.$ $\left.\mathrm{x} 10^{4} \mathrm{M}^{-1} \mathrm{~cm}^{-1}\right)$ : the assay mixture contained $2 \mathrm{mM}$ ABTS, $0.1 \mathrm{M}$ Na-citrate buffer, $\mathrm{pH}$ 3.0.

\section{In situ exfoliation of graphite}

Graphite powder $1 \mathrm{mg} / \mathrm{mL}$ (Aldrich, 332461, mesh number of grains $+100,>75 \%$ ) was exfoliated in batches

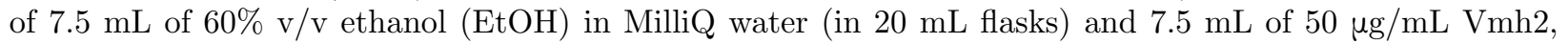
using a medium power tip sonicator (Q125 Sonicator, QSonica, $125 \mathrm{~W}, 20 \mathrm{kHz}$, inbuilt power meter power output, $19 \mathrm{~W}$ ) and cooling the system in an ice bath. The exfoliation was stopped when the energy value was $450 \mathrm{KJ}$. To remove the unexfoliated material, controlled centrifugation was performed for 40 minutes at different speed $(40 \mathrm{~g}, 160 \mathrm{~g}$ and $620 \mathrm{~g})$. Three types of experiments were tested with the laccase enzymes:

-A 7-ml solution of PoxA1b or Lac-Vmh2 in 40\% EtOH was added to Vmh2-exfoliated graphene and incubated at $4^{\circ} \mathrm{C}$ whilst stirring continuously.

-The immobilization was performed by adding the wild-type or chimeric enzyme solution to graphite powder at the beginning of the exfoliation.

-The wild-type or chimeric enzyme solution was added during the last 10 minutes of exfoliation. The process was performed normalizing the activity units $\left(4 \mathrm{U}_{\text {tot }}\right.$ for both) between wild-type POXA1b and Lac-Vmh2 (0.16 $\mathrm{mg}$ and $0.44 \mathrm{mg}$, respectively).

\section{RESULTS AND DISCUSSION}

\section{Laccase immobilization on FLG}

Graphite exfoliation was carried out, as previously described by exposing mixtures of Vmh2 protein and graphite to ultrasonic waves. (Gravagnuolo et al., 2015) Vmh2-exfoliated graphene is generally stable in $60 \% \mathrm{EtOH}$ thanks to the presence of the HFB. On the other hand, enzymes are usually used and stable in aqueous buffers. Thus, conditions have to be assessed to preserve both the stability of graphene dispersion and the enzyme activity. The wild-type PoxA1b laccase and Lac-Vmh2 were dialyzed toward different ethanol concentration $(20 \%, 40 \%, 50 \%$ and $60 \% \mathrm{EtOH}$,) and $10 \mathrm{mM}$ Tris- $\mathrm{HCl} \mathrm{pH} \mathrm{8,} \mathrm{to} \mathrm{test} \mathrm{the} \mathrm{stability} \mathrm{of} \mathrm{the} \mathrm{enzyme}$ in these conditions. Concomitantly, the Vmh2-graphene samples in $60 \% \mathrm{EtOH}$ were centrifuged and pellets were solubilized with or without the addition of $0.05 \mathrm{mg} \mathrm{mL}^{-1}$ of $\mathrm{Vmh} 2$ in the same conditions tested for enzymatic stability. The optimal condition for the graphene stability was 50\% EtOH $+\mathrm{Vmh} 2$ and $40 \% \mathrm{EtOH}$ + Vmh2 (Figure S1). On the other hand, the enzyme stability was reasonable up to $40 \%$ EtOH (Table S1). Thus, looking both at the enzyme and the graphene stability, the $40 \% \mathrm{EtOH}$ was selected as the optimal solvent for graphite exfoliation in the presence of laccase.

To study and optimize the condition of laccase immobilization on the FLG, several tests were performed (Figure 1). Addition of the enzymes (wild-type or chimera) was performed after exfoliation of graphite in the presence of $\mathrm{Vmh} 2$ (route A). Addition of the enzyme solutions to graphite powder was performed at the beginning of the exfoliation (route B). Or, in situ exfoliation of graphite with Vmh2 was followed by the addition of the enzymes in the last step of sonication (route C). Each route was compared by measuring the enzymatic activity (Pezzella et al., 2017) of the biofunctionalized FLG (previously separated after centrifugation at 13,000rpm for 15 minutes) obtained after centrifugation. Results are displayed in table S2. Route A lead to final no enzymatic activity, indicating that negligible amounts of enzymes can be immobilized on FLG after exfoliation of graphite with Vmh2. When the chimera was used to exfoliate graphene from graphite (route B), the extensive ultrasonication time lead to a complete loss of the enzymatic activity of laccase. To avoid a long exposure of the enzymes to ultrasonic waves, the route $\mathrm{C}$ was used in the course of this work. In the latter route, wild type or chimera were added during the last 10 minutes 
of exfoliation process in order to prevent enzyme inactivation. An immobilization yield of 5\% and $11 \%$, for PoxA1b or Lac-Vmh2 was respectively estimated considering the enzymatic activity before and after immobilization. According to the activity of the immobilized Lac-Vmh2, this corresponds to an enzyme loading of about $0.4 \mathrm{U}$ per mg of FLG (Table 1). Lac-Vmh2 showed a slight increased amount of attached enzyme as compared to the wild-type enzyme. Indeed, POXA1b was able to stick to graphene, as already observed using polystyrene (Sorrentino et al., 2019). Nevertheless, the stability of the biofunctionalized graphene obtained with Lac-Vmh2 is higher than that of the wild-type enzyme, in terms of both activity and adhesion (Table 1).

Table 1. Summary of the sample activity and their characteristics

\begin{tabular}{llll}
\hline Samples & Units on $\mathrm{mg}$ of graphene $(\mathrm{U} / \mathrm{mg})$ & $\mathrm{t}_{1 / 2}$ (days) & Activity after washing \\
\hline Graphene/PoxA1b & $0.3 \pm 0.1$ & 17 & stable up to the 2nd washes \\
Graphene/Lac-Vmh2 & $0.4 \pm 0.1$ & 26 & stable up to the 4 th washes \\
\hline
\end{tabular}

The biofunctionalized graphene was stable at least for 30 days (Figure 2A). Raman spectroscopy was also performed in order to characterize the number of graphene layers for these biofunctionalized FLG (Figure 2B). According to Ferrari's work (Ferrari et al., 2006), the high-energy band observed at $2700 \mathrm{~cm}^{-1}$ stems for the presence of only few graphene layers, as already observed in the case of the native Vmh2 hydrophobin. (Gravagnuolo et al., 2015) It is noteworthy that the Raman spectrum was also performed after several months on the same stock solution of Lac-Vmh2-functionalized FLG without showing any restacking nor aggregation phenomena.

\section{Exploitation of biofunctionalized FLG in electrochemical biosensing}

GC electrodes were then modified by drop-casting a solution of biofunctionalized FLG. The incubation was performed at room temperature until the electrode was completely dry and several washes with citratephosphate buffer $\mathrm{pH} 5.0$ were executed to eliminate unbound sample.

Figure 3 displays a typical SEM image of biofunctionalized FLG deposited onto planar gold electrode. This underlines the homogenous dispersion of FLG both in solution and at the surface of the electrode. According to size distribution study, the size of biofunctionalized FLG are mostly below $2 \mu \mathrm{m}^{2}$. These nanostructured bioelectrodes were employed for biosensing experiments. The principle of laccase biosensors is based on the enzymatic oxidation of phenols or $o$-diphenols into quinones, the latter being subsequently reduced at the nanostructured electrode polarized at a redox potential required for the electroreduction of quinone into phenols, i.e. $\mathrm{E}=-0.2 \mathrm{~V}$ vs. SCE. The regeneration of the catechol derivative triggers an amplification cycle of "enzymatic oxidation/electrochemical reduction" which increase biosensing sensitivity.

Addition of both catechol and dopamine was monitored at Lac-Vmh2- biofunctionalized FLG electrodes. Figure 4A displays a representative chronoamperometry experiment performed upon addition of catechol.

Chronoamperometry at different volumes of FLG (Figure 4B) were performed showing that an optimum value of biofunctionalized FLG is reached after the drop-coating of $80 \mu \mathrm{L}(16 \mathrm{mU})$ solution on GC electrodes. These experiments underline the fact that an optimal volume of FLG is needed in order to maximize the amount of immobilized enzymes while also providing efficient diffusion of catechol into the nanostructured FLG conductive film. In order to indirectly confirm the interaction between the Vmh2 domain of the chimera and graphene layer, the wild-type POXA1b was also used in exfoliation process of FLG. However, negligible current was observed for catechol oxidation, underlining the fact less adsorption of POXA1b was observed on FLG. This also demonstrates the important role of the Vmh2 domain in order to immobilize laccase at the surface of FLG.

Figure 5 displays the calibration curves for catechol and dopamine recorded at these electrodes. The shape of the curves is governed by the enzymatic reaction which is reliability modelized according to a typical Michaelis-Menten kinetics. Table 2 summarizes the electrochemical characteristics of the modelized curves. 
Table 2. Electrochemical characteristics obtained after modelization of the curve according MichaelisMenten's kinetics

\begin{tabular}{llll}
\hline & $\mathrm{K}_{\mathrm{M}}(\mathrm{mM})$ & $\operatorname{Imax}\left(\mathrm{nA} \mathrm{cm}^{-2}\right)$ & $\mathrm{R}^{2}$ \\
\hline Catechol & $\mathbf{1 . 1}$ & $\mathbf{7 7 5 . 7}$ & $\mathbf{0 . 9 9}$ \\
Dopamine & $\mathbf{3 . 0}$ & $\mathbf{5 5 . 5}$ & $\mathbf{0 . 9 5}$ \\
\hline
\end{tabular}

The apparent Michaelis-Menten constant $\left(\mathrm{K}_{\mathrm{Mapp}}\right)$ reflects the enzyme-substrate affinity which is, as expected, higher in the case of dopamine as compared to catechol for laccase.

The linear part of the curve is shown in the Figure 6. While LOD of $20 \mu \mathrm{M}$ was measured for both catechol and dopamine respectively, sensitivity of $0.27 \mathrm{~mA} \mathrm{M}^{-1} \mathrm{~cm}^{-2}\left(\mathrm{R}^{2}=0.97\right)$ towards catechol and $16.4 \mu \mathrm{A} \mathrm{M}^{-1}$ $\mathrm{cm}^{-2}\left(\mathrm{R}^{2}=0.96\right)$ towards dopamine were measured with respective linear range of 20 to $1000 \mu \mathrm{M}$ and 20 to $250 \mu \mathrm{M}$. While the most efficient phenolic biosensors are based on a combination of tyrosinases and laccases, (S. Freire, Thongngamdee, Durán, Wang, \& T. Kubota, 2002; Yaropolov, Kharybin, Emnéus, Marko-Varga, \& Gorton, 1995) or based on the use of redox hydrogels (Ferry \& Leech, 2005; Rodríguez-Delgado et al., 2015), this type of nanostructured biosensor approaches the performances of other types of biosensors associating graphene and laccases (Boujakhrout et al., 2016; Palanisamy et al., 2017; Rodríguez-Delgado et al., 2015)

\section{CONCLUSION}

This work shows that Lac-Vmh2 chimera enzyme can be used both as a surfactant of FLG while also providing enzymatic activity to the biofunctionalized nanomaterials. Deposition of these biofunctionalized FLG on electrodes affords the fabrication of catechol and dopamine biosensors. This novel strategy of functionalizing carbon nanomaterials with specific chimeric enzymes paves the way for the development of many types of novel chimeric enzymes which can be developed for a variety of applications involving multienzymatic systems and biofunctionalization of nanomaterials.

\section{ACKNOWLEDGMENT}

This project is funded by Italian Education, University and Research Ministry (MIUR), French National Research Agency (ANR) and co-funded by European Union's Horizon 2020 research and innovation program under the framework of ERA-NET Cofund MarTERA (Maritime and Marine Technologies for a new Era). This work was supported by the Agence Nationale de la Recherche through the LabEx ARCANE programe (ANR-11-LABX-0003-01) and the Graduate School on Chemistry, Biology and Health of Univ Grenoble Alpes CBH-EUR-GS (ANR-17-EURE-0003). The authors acknowledge support from the plateforme de Chimie NanoBio ICMG FR 2607 (PCN-ICMG).

\section{REFERENCES}

Boujakhrout, A., Jimenez-Falcao, S., Martínez-Ruiz, P., Sánchez, A., Díez, P., Pingarrón, J. M., \& Villalonga, R. (2016). Novel reduced graphene oxide-glycol chitosan nanohybrid for the assembly of an amperometric enzyme biosensor for phenols. Analyst ,141 (13), 4162-4169. https://doi.org/10.1039/C5AN02640G

B. Ramakrishna, T. R., D. Nalder, T., Yang, W., N. Marshall, S., \& J. Barrow, C. (2018). Controlling enzyme function through immobilisation on graphene, graphene derivatives and other two dimensional nanomaterials. Journal of Materials Chemistry B , 6 (20), 3200-3218. https://doi.org/10.1039/C8TB00313K

Brownson, D. A. C., Lacombe, A. C., Gomez-Mingot, M., \& Banks, C. E. (2011). Graphene oxide gives rise to unique and intriguing voltammetry.RSC Advances , 2 (2), 665-668. https://doi.org/10.1039/C1RA00743B

Brownson, D. A. C., Munro, L. J., Kampouris, D. K., \& Banks, C. E. (2011). Electrochemistry of graphene : Not such a beneficial electrode material? RSC Advances , 1 (6), 978-988. https://doi.org/10.1039/C1RA00393C 
C. Ferrari, A., Bonaccorso, F., Fal'ko, V., S. Novoselov, K., Roche, S., Boggild, P., .. Kinaret, J. (2015). Science and technology roadmap for graphene, related two-dimensional crystals, and hybrid systems. Nanoscale , 7 (11), 4598-4810. https://doi.org/10.1039/C4NR01600A

C. Rodrigues, R., Ortiz, C., Berenguer-Murcia, A., Torres, R., \& Fernandez-Lafuente, R. (2013). Modifying enzyme activity and selectivity by immobilization. Chemical Society Reviews , 42 (15), 6290-6307. https://doi.org/10.1039/C2CS35231A

Ferrari, A. C., Meyer, J. C., Scardaci, V., Casiraghi, C., Lazzeri, M., Mauri, F., .. Geim, A. K. (2006). Raman Spectrum of Graphene and Graphene Layers. Physical Review Letters , 97 (18), 187401. https://doi.org/10.1103/PhysRevLett.97.187401

Ferry, Y., \& Leech, D. (2005). Amperometric Detection of Catecholamine Neurotransmitters Using Electrocatalytic Substrate Recycling at a Laccase Electrode. Electroanalysis , 17 (2), 113-119. https://doi.org/10.1002/elan.200403069

Fritea, L., Le Goff, A., Putaux, J.-L., Tertis, M., Cristea, C., Sandulescu, R., \& Cosnier, S. (2015). Design of a reduced-graphene-oxide composite electrode from an electropolymerizable graphene aqueous dispersion using a cyclodextrin-pyrrole monomer. Application to dopamine biosensing. Electrochimica Acta ,178 , 108-112. https://doi.org/10.1016/j.electacta.2015.07.124

Fritea, L., Tertis, M., Sandulescu, R., \& Cristea, C. (2018). Chapter Eleven-Enzyme-Graphene Platforms for Electrochemical Biosensor Design With Biomedical Applications. In C. V. Kumar (Ed.), Methods in Enzymology (p. 293-333). https://doi.org/10.1016/bs.mie.2018.05.010

Gravagnuolo, A. M., Morales-Narvaez, E., Longobardi, S., da Silva, E. T., Giardina, P., \& Merkoci, A. (2015). In Situ Production of Biofunctionalized Few-Layer Defect-Free Microsheets of Graphene.Advanced Functional Materials , 25 (18), 2771-2779. https://doi.org/10.1002/adfm.201500016

Kampouris, D. K., \& Banks, C. E. (2010). Exploring the physicoelectrochemical properties of graphene. Chemical Communications , 46 (47), 8986-8988. https://doi.org/10.1039/C0CC02860F

Keeley, G. P., O'Neill, A., Holzinger, M., Cosnier, S., Coleman, J. N., \& Duesberg, G. S. (2011). DMFexfoliated graphene for electrochemical NADH detection . 7747-7750.

Lalaoui, N., Le Goff, A., Holzinger, M., Mermoux, M., \& Cosnier, S. (2015). Wiring Laccase on Covalently Modified Graphene : Carbon Nanotube Assemblies for the Direct Bio-electrocatalytic Reduction of Oxygen. Chemistry - A European Journal , 21 (8), 3198-3201. https://doi.org/10.1002/chem.201405557

Le Goff, A., Reuillard, B., \& Cosnier, S. (2013). A Pyrene-Substituted Tris(bipyridine)osmium(II) Complex as a Versatile Redox Probe for Characterizing and Functionalizing Carbon Nanotube- and Graphene-Based Electrodes. Langmuir, 8736-8742.

Lotya, M., Hernandez, Y., King, P. J., Smith, R. J., Nicolosi, V., Karlsson, L. S., .. C Coleman, J. N. (2009). Liquid Phase Production of Graphene by Exfoliation of Graphite in Surfactant/Water Solutions. Journal of the American Chemical Society , 131 (10), 3611-3620. https://doi.org/10.1021/ja807449u

Novoselov, K. S., Fal'ko, V. I., Colombo, L., Gellert, P. R., Schwab, M. G., \& Kim, K. (2012). A roadmap for graphene. Nature ,490 (7419), 192-200. https://doi.org/10.1038/nature11458

Palanisamy, S., Ramaraj, S. K., Chen, S.-M., Yang, T. C. K., Yi-Fan, P., Chen, T.-W., ... Selvam, S. (2017). A novel Laccase Biosensor based on Laccase immobilized Graphene-Cellulose Microfiber Composite modified Screen-Printed Carbon Electrode for Sensitive Determination of Catechol.Scientific Reports , 7 (1), 1-12. https://doi.org/10.1038/srep41214

Pezzella, C., Giacobelli, V. G., Lettera, V., Olivieri, G., Cicatiello, P., Sannia, G., \& Piscitelli, A. (2017). A step forward in laccase exploitation : Recombinant production and evaluation of techno-economic feasibility of the process. Journal of Biotechnology , 259 , 175-181. https://doi.org/10.1016/j.jbiotec.2017.07.022 
Pezzella, C., Guarino, L., \& Piscitelli, A. (2015). How to enjoy laccases. Cellular and Molecular Life Sciences , 72 (5), 923-940. https://doi.org/10.1007/s00018-014-1823-9

Piscitelli, A., Pennacchio, A., Longobardi, S., Velotta, R., \& Giardina, P. (2017). Vmh2 hydrophobin as a tool for the development of "self-immobilizing" enzymes for biosensing. Biotechnology and Bioengineering, 114 (1), 46-52. https://doi.org/10.1002/bit.26049

Pumera, M. (2011). Graphene in biosensing. Materials Today ,14 (7), 308-315. https://doi.org/10.1016/S1369-7021(11)70160-2

Pumera, M. (2012). Voltammetry of carbon nanotubes and graphenes : Excitement, disappointment, and reality. The Chemical Record ,12 (1), 201-213. https://doi.org/10.1002/tcr.201100027

Rodriguez-Delgado, M. M., Aleman-Nava, G. S., Rodriguez-Delgado, J. M., Dieck-Assad, G., Martinez-Chapa, S. O., Barcelo, D., \& Parra, R. (2015). Laccase-based biosensors for detection of phenolic compounds. TrAC Trends in Analytical Chemistry , 74 (Supplement C), 21-45. https://doi.org/10.1016/j.trac.2015.05.008

S. Freire, R., Thongngamdee, S., Duran, N., Wang, J., \& T. Kubota, L. (2002). Mixed enzyme (laccase/tyrosinase)-based remote electrochemical biosensor for monitoring phenolic compounds. Analyst ,127 (2), 258-261. https://doi.org/10.1039/B110011D

Smith, R. J., Lotya, M., \& Coleman, J. N. (2010). The importance of repulsive potential barriers for the dispersion of graphene using surfactants. New Journal of Physics , 12 (12), 125008. https://doi.org/10.1088/1367-2630/12/12/125008

Sorrentino, I., Giardina, P., \& Piscitelli, A. (2019). Development of a biosensing platform based on a laccase-hydrophobin chimera.Applied Microbiology and Biotechnology , 103 (7), 3061-3071. https://doi.org/10.1007/s00253-019-09678-2

Yaropolov, A. I., Kharybin, A. N., Emneus, J., Marko-Varga, G., \& Gorton, L. (1995). Flow-injection analysis of phenols at a graphite electrode modified with co-immobilised laccase and tyrosinase.Analytica Chimica Acta , 308 (1), 137-144. https://doi.org/10.1016/0003-2670(94)00404-A

Zampieri, F., Wosten, H. A. B., \& Scholtmeijer, K. (2010). Creating Surface Properties Using a Palette of Hydrophobins. Materials ,3 (9), 4607-4625. https://doi.org/10.3390/ma3094607

Zhang, W., Jia, B., \& Furumai, H. (2018). Fabrication of graphene film composite electrochemical biosensor as a pre-screening algal toxin detection tool in the event of water contamination. Scientific Reports , 8 (1), 1-10. https://doi.org/10.1038/s41598-018-28959-w

\section{FIGURE LEGENDS}

Figure 1. Biofunctionalization process of FLG with Vmh2-Lac

Figure 2 . (A) Stability of graphene dispersion at time zero and after 35 days: lane1 PoxA1b-exfoliated graphene; lane 2 Lac-Vmh2-exfoliated graphene ; (B) Representative Raman spectrum of a Vmh2-Lacbiofunctionlized FLG film.

Figure 3 . SEM image of biofunctionalized FLG at the surface of a gold electrode; (inset) size distribution of biofunctionalized FLG obtained by ImageJ.

Figure 4. (A) Chronoamperometry performed at Lac-Vmh2-biofunctionlized FLG electrode after successive additions of catechol (indicated by the arrows, applied potential $=-0.2 \mathrm{~V}$ vs. SCE, $0.1 \mathrm{M} \mathrm{PBS,} \mathrm{pH} 6,25$ $\operatorname{deg}$ ); (B) Plot of the maximum current towards volume of drop-coated Lac-Vmh2-biofunctionlized FLG solutions.

Figure 5. Plot of the catalytic current towards increasing concentrations of () catechol and ()dopamineforelectrodes(MeasurementsperformedbychronoamperometryatE = 
-0.2 Vvs.SCE, 0.1MPBS, pH6, 25degC)

Figure 6. Plot of the linear part of the catalytic current density towards concentration of (A) catechol and (B) dopamine ( $\mathrm{E}_{\text {applied }}=-0.2 \mathrm{~V}$ vs. SCE, 0.1 M PBS, pH 6, $25 \operatorname{degC}$ ).

\section{FIGURES}

Figure 1

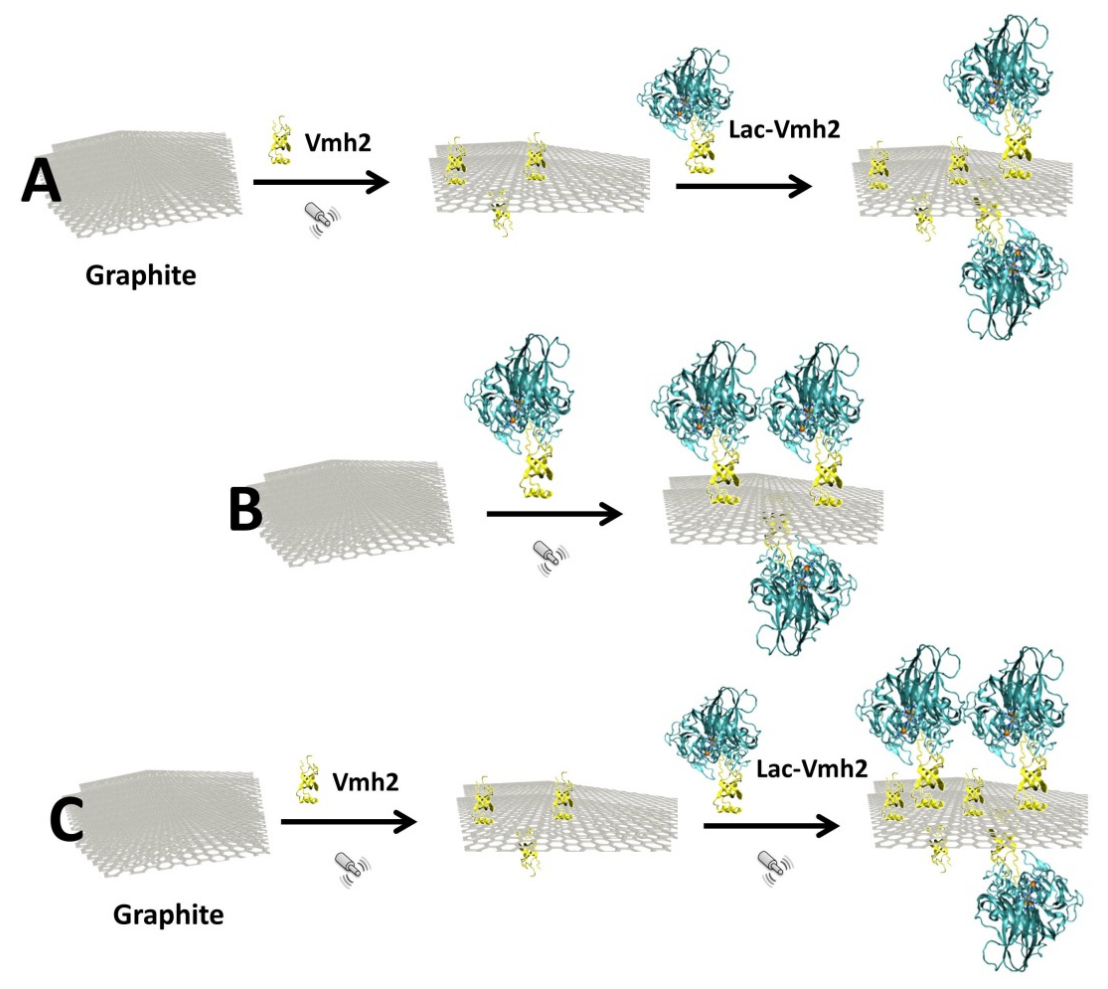

Figure 2
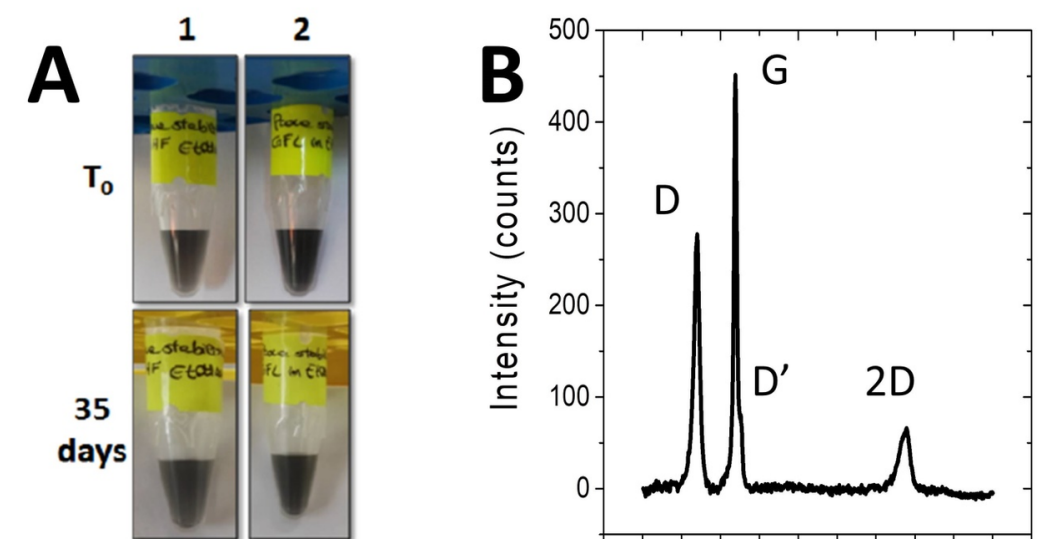

Figure 3 


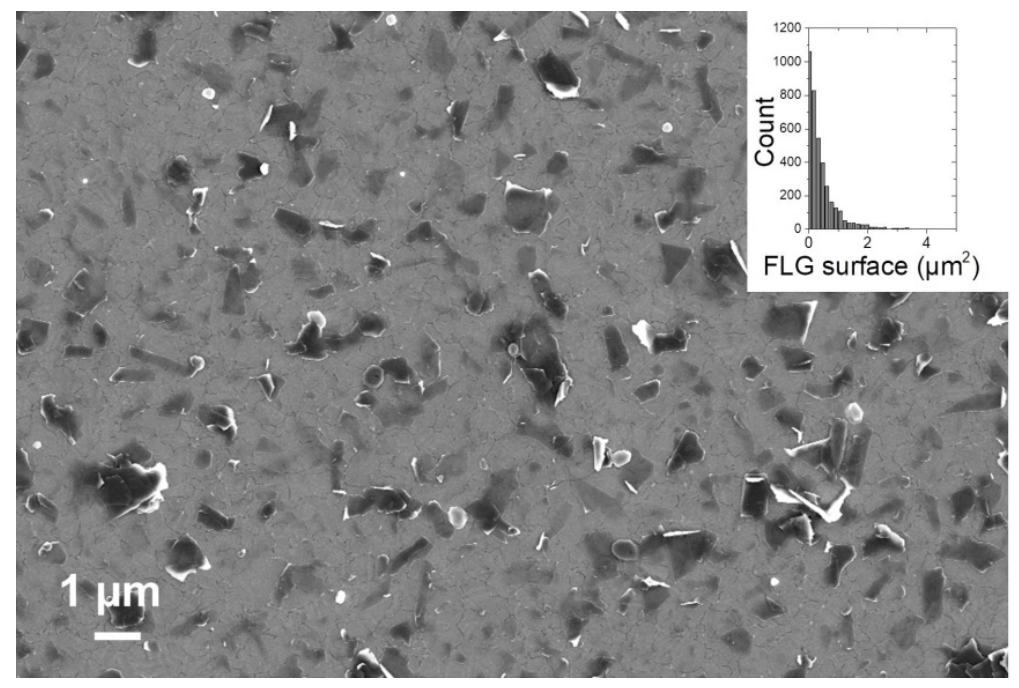

Figure 4 .

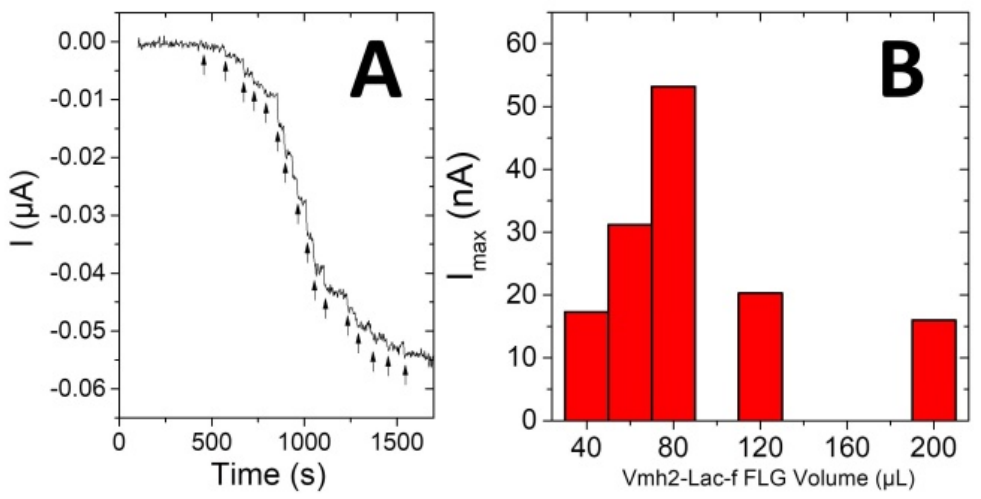

Figure 5. 


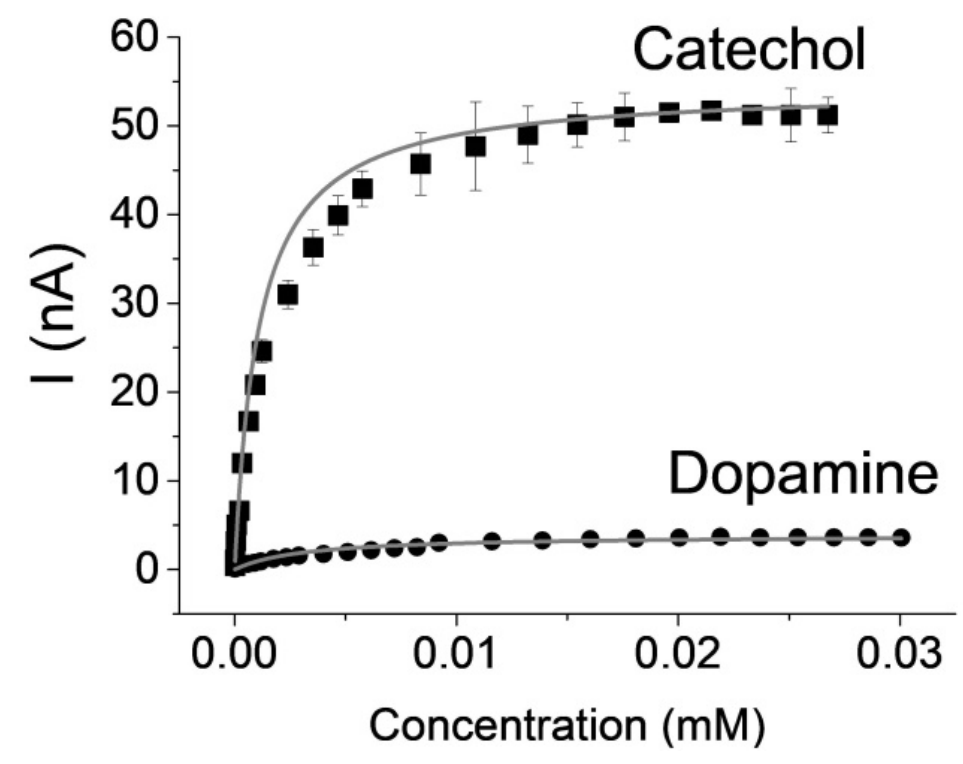

Figure 6.

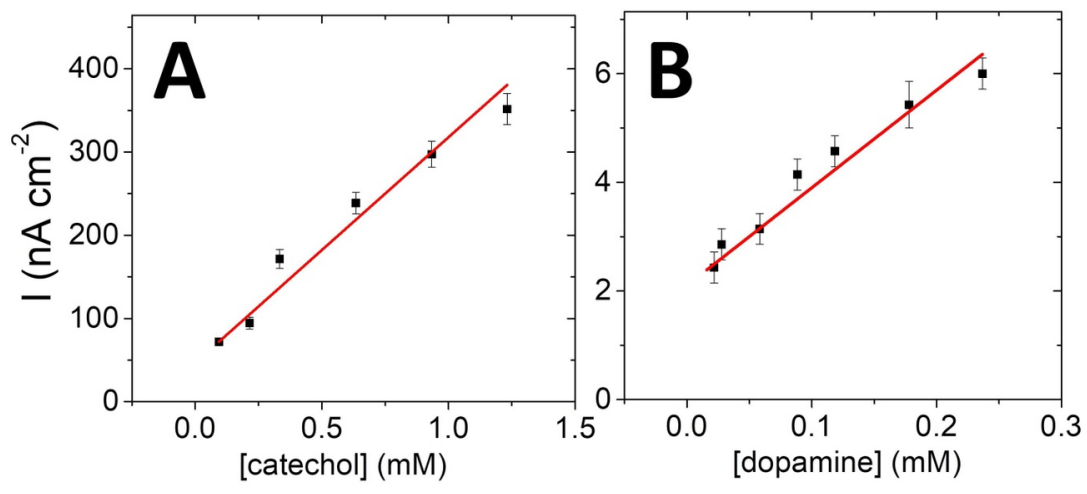



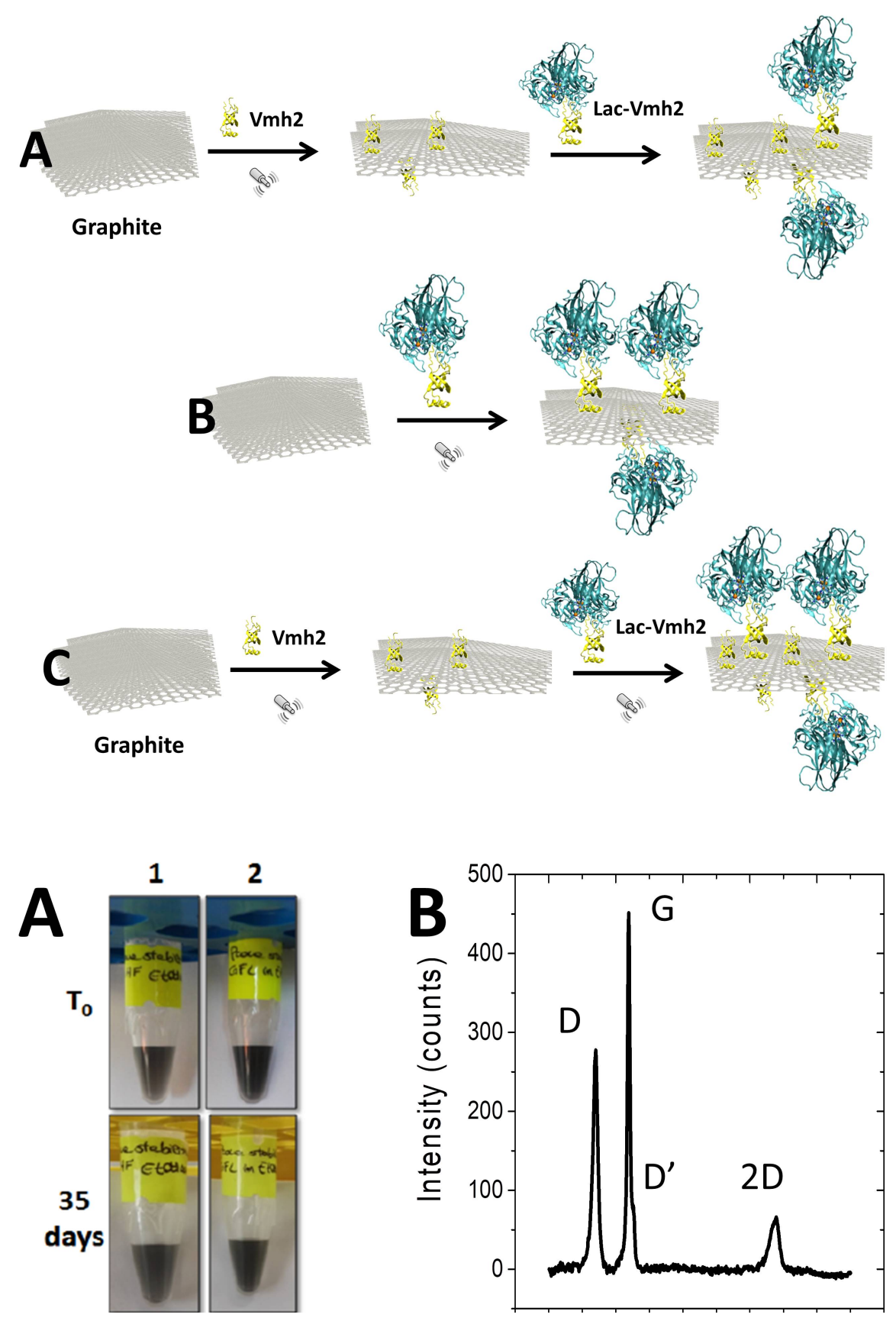

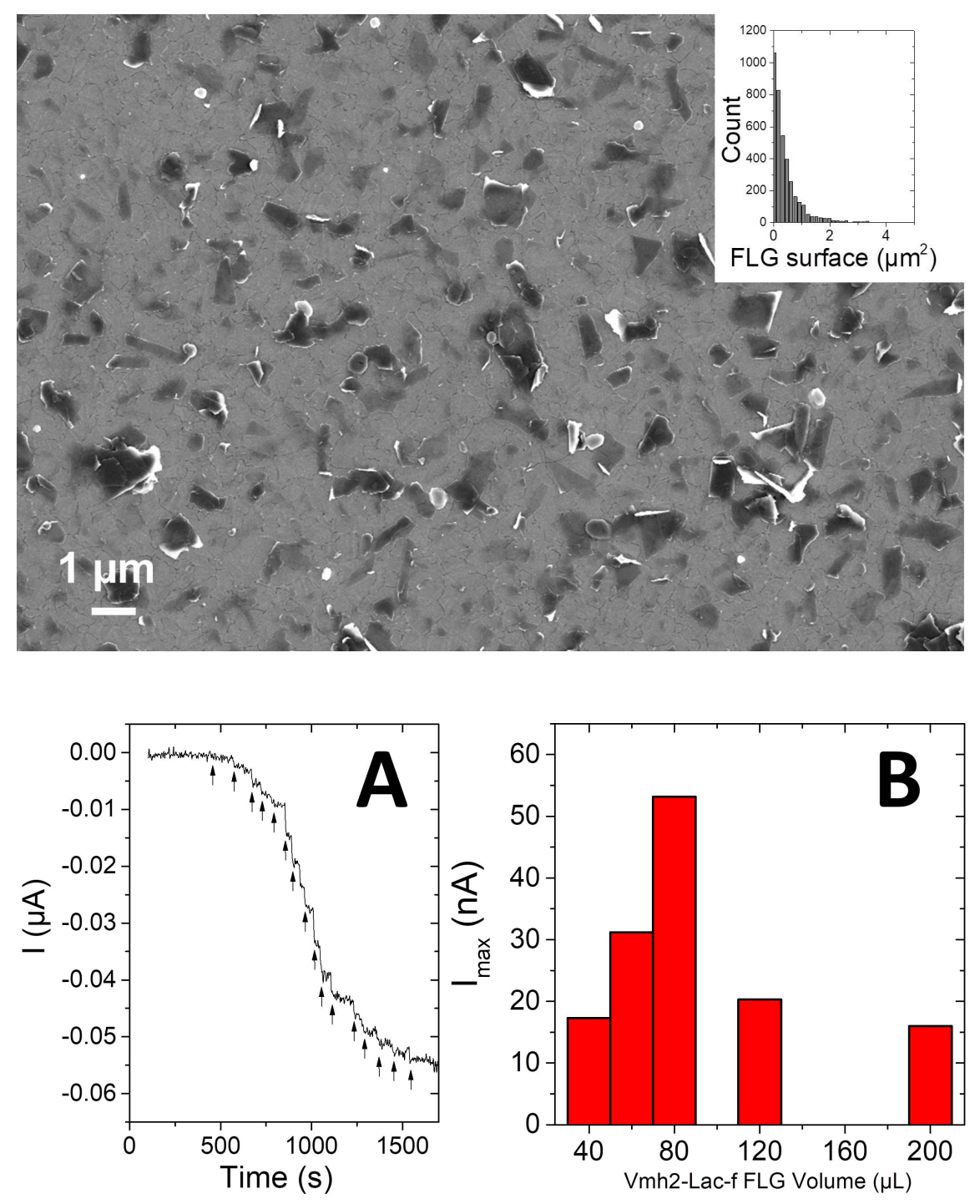

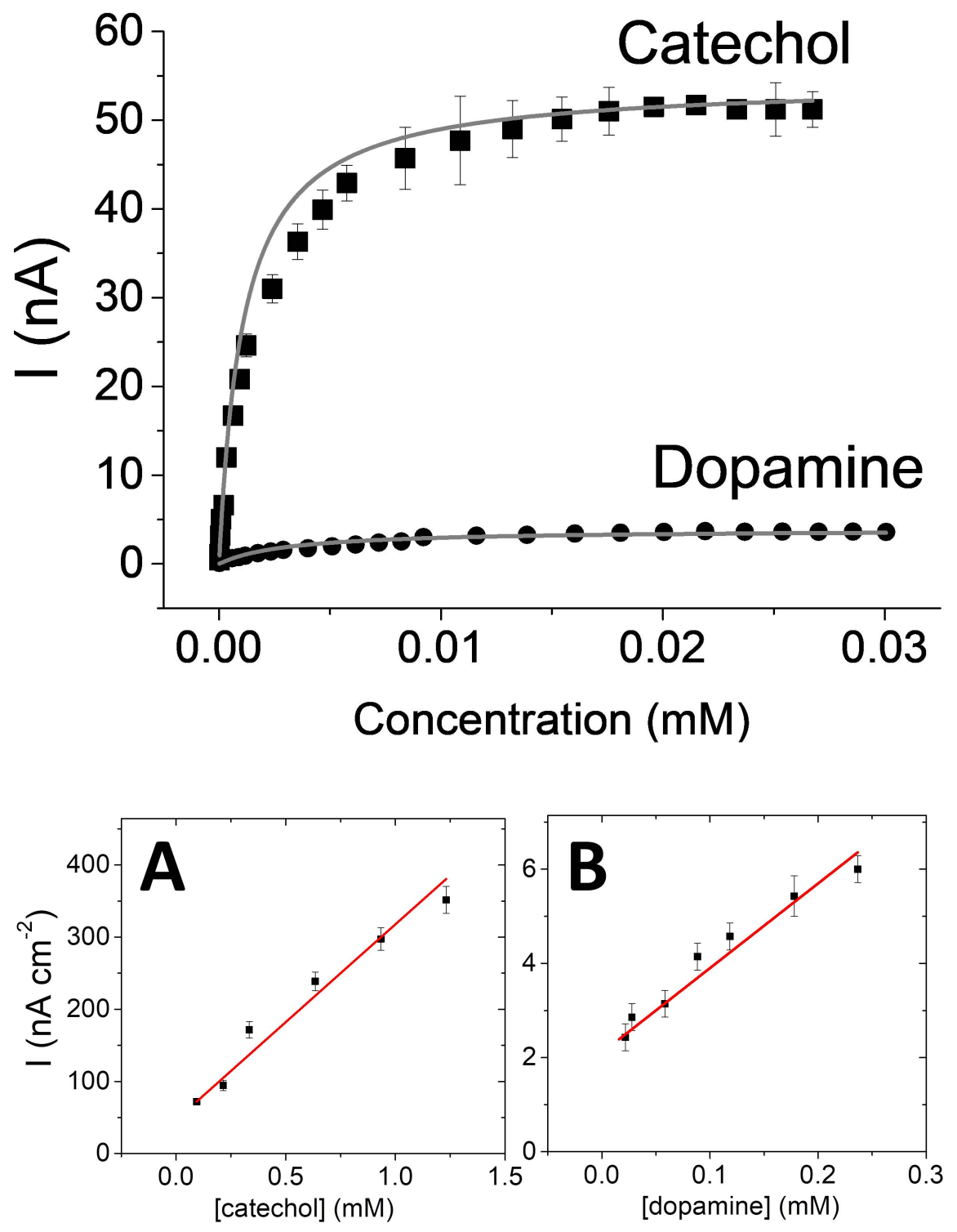